\title{
Artikel
}

Burkhard Meyer-Sickendiek*

\section{Die Satire als invektive Gattung}


Abstract: A discussion of satire as borderline case of invectivity will be presented in this paper. The particular focus lies on literary debates in eighteenth-century Britain and in Germany. British satirists like Dryden, Haywood or Pope described ridicule and sarcasm as main features of satire, however, it was viewed as necessary to uphold the distinction between satire and libel resp. lampoon. This distinction was explained by concepts of urban wit or raillery. In German literature Wieland introduced the concept of wit in his satirical writings, however, since romanticism it was replaced with the opposition between sarcasm and ,Humor'.

Keywords: Satire, Spott, Sarkasmus, Witz, Stichelei, Humor - satire, ridicule, sarcasm, lampoon, raillery, humour

*PD Dr. Burkhard Meyer-Sickendiek, FU Berlin, bumesi@zedat.fu-berlin.de

Wohl kaum ein poetisches literarisches Genre ist für eine Erörterung dessen, was unter Invektivität zu verstehen ist, so einschlägig wie die Gattung der Satire. Orientiert man sich dabei etymologisch, dann zeigt der Begriff der Satire in all seiner Bedeutungsvielfalt, wie sehr das satirische Sprechen seit der Neuzeit als Grenzbereich des Invektiven gelten kann. Anders gesagt: Was Invektivität bezeichnet, lässt sich ex negativo auch durch die Grenzziehung zur bzw. die Integration der Satire recht genau bestimmen. Doch zunächst zur Begriffsgeschichte: Der Begriff ,Satire' stammt vom Lateinischen ,satura', welches sich herleiten lässt aus dem Begriff ,lanx satura', was als ,Opferschüssel mit verschiedenen Früchten' übersetzbar ist und die Satire seit Ennius als ein ,Erfülltsein von mannigfaltigen Bestandteilen' definiert. Zwar galt die Satire zumindest nach römischem Verständnis als römische Schöpfung, denn von Quintilian stammt der auf Horaz und Juvenal bezogene Satz "satura quidem tota nostra est." ${ }^{\text {B }}$ Bis in die Renaissance wurde Satire jedoch fälschlicherweise auch vom griechischen ,Satyr', also dem Begleiter des Dionysos, abgeleitet sowie vom Satyrspiel, also der burlesken Parodie im Anschluss an eine Tragö-

1 Quintilian (2000) Institutio oratoria 10, 1, 93. dientrilogie. ${ }^{2}$ Erst mit der etymologischen Unterscheidung zwischen Satyr und Satura, wie sie Isaac Casaubon in De satyrica graecorum poesi et romanorum satira libri duo vornahm, wurde die Satire unter Bezug auf die römische Tradition identifizierbar als eine entrüstete und insofern didaktisch-moralische Verspottung des Lasters. Sie gewann ihren moralischen Anspruch durch die Kanonisierung der römischen Verssatire, da mit dieser die Verspottung realer Personen im Sinne des Pasquills bzw. der Polemik aus der satirischen Indignation ausgeschlossen wurde. ${ }^{3}$

Die Satire wurde dadurch zum klassischen Medium ironischen Tadelns durch falsches Lob: Sie kennt also sowohl die Verstellung (dissimulatio) und zwar im Sinne des humorvollen "ridentem dicere verum" (lachend das Wahre sagen, Horaz) als auch die Verspottung (illusio), dieses dient jedoch im Rahmen der ,indignatio' (Entrüstung, Juvenal) der satirischen Kritik an einer lasterhaften oder verkehrten Welt. ${ }^{4}$ Nach Arntzen lässt sich die Satire daher auch als eine Tadelrede begreifen, ${ }^{5}$ welche wiederum in eine moralische und in eine prophetische zu unterteilen ist. Die

2 Vgl. dazu: Becker-Cantarino (1985) Satire.

3 Vgl. Brummack (1977) Satire.

4 Vgl. Meyer-Sickendiek (2007) Satire, S. 447.

5 Vgl. Arntzen (2003) Satire. 
prophetische Tadelrede gehe von einer göttlichen Botschaft aus, weshalb deren Verfasser im Namen Gottes gegen das sündige Volk spreche. Die Normen sind nicht gesellschaftlicher oder ethischmoralischer Natur, sondern die offenbarten Gebote Gottes. Diese Tadelrede zielt auf Buße, auf Umkehr des Einzelnen und auf moralische Besserung der Gesellschaft ab: Man denke an Sebastian Brants Narrenschiff. Dagegen zielt die moralische Tadelrede auf die Moral einer Geschichte $a b$, welche die Besserung eines Zustands oder einer Verhaltensweise beabsichtigt: Prominentester Repräsentant dieser Form der Tadelrede ist die römische Verssatire Juvenals. Wenn Schiller den „Widerspruch der Wirklichkeit mit dem Ideale"6 zum Gegenstand des Satirikers erklärt, wenn Hegel das "Wissen und Wollen des Guten und der Tugend" als Grund dafür nennt, satirisch "in einen feindlichen Gegensatz gegen das Verderben seiner Gegenwart zu treten", 7 dann gehen diese Definitionen maßgeblich auf die Indignation Juvenals zurück. Die moralische Entrüstung des Satirikers ist nach Arntzen jedoch von einer rein psychischen Befindlichkeit zu unterscheiden, da sie der Produzent der literarischen Darstellung, also eine genuin literarische Entrüstung ist.

Für eine genauere Analyse und Beschreibung dieser Zusammenhänge kann eine Formel Brummacks herangezogen werden. Brummack definiert die Satire als eine "ästhetisch sozialisierte Aggression", betont also drei Konstituenten: eine psychologische, eine soziale und eine ästhetische. ${ }^{8}$ Die psychologische Dimension bedenkt die aggressive Haltung des Satirikers zum Gegenstand der Satire, wobei nach Brummack zwei Grundformen zu unterscheiden sind: Die heitere Satire als Verspottung der Torheiten, welche auf Horaz, und die strafende Satire als Verspottung des Lasterhaften, welche auf Juvenal zurückgeht. Hat die Satire diesen Angriffscharakter mit der Polemik und der Groteske gemein, so unterscheidet sie sich jedoch von der ersten Form durch ihre geringere Vulneranz, von der zweiten durch ihren Wirklichkeits- bzw. Gesellschaftsbezug. Nur die rhetorische Aggression des Polemikers ist mit der körperlichen Aggression vergleichbar, da dem Angegriffenen eine der körperlichen Gewalt vergleichbare soziale

6 Schiller (1962) Dichtung, S. 721f.

7 Hegel (2013) Vorlesungen über die Ästhetik II, S. 122.

8 Brummack (1971) Satire, S. 282.
Schädigung durch sarkastische Verspottung droht: Dies geschieht in der Satire nach Maßgabe inrer Poetik nicht. Und im Unterschied zur Groteske richtet sich das aggressive Verlachen nach der Poetik der Satire auf die Gesellschaft und deren lasterhaften Zustand, wohingegen eine solche Referentialität in der Groteske nicht unbedingt auszumachen ist. Die Groteske ist also „keineswegs gegen eine bestimmte Seinsform der Natur der Gesellschaft und des individuellen menschlichen Daseins gerichtet", wie Tschizewskij betonte. ${ }^{9}$

Zwar finden beide Formen vor allem in Zeiten der Krise ihren Ausdruck, was etwa die Gattung der satirischen Simpliziade und des grotesken Grobianismus in der Zeit des 30-jährigen Krieges erklärt. Der von Gaier formulierte Merksatz "Satire ist sprachliche Auseinandersetzung mit einer bedrohlichen Wirklichkeit"10 - suggeriert jedoch, dass der Satiriker im Unterschied zum Künstler der Groteske diese Bedrohung durch Übertragung oder Einschränkung zum definierten satirischen Angriffsziel umformt. Diese Auseinandersetzung mit der Wirklichkeit ist nach Gaier zudem eine Destruktion der Realität, ${ }^{11}$ wobei dies die gegenbildliche Funktion der Gattung betont. Der Begriff der Gegenbildlichkeit bezeichnet das für die satirische Fiktion grundlegende Motiv der verkehrten Welt, die als Zerrspiegel der Realität deren Entlarvung und Bloßstellung bewirkt. Auch Arntzen nennt die Negativität, das ,Verkehrte' als wichtigstes Gattungsmerkmal: In der Satire wird das ,Verkehrte' so dargestellt, dass dessen Abschaffung gefordert wird. Insgesamt sind es nach Arntzen also vier Elemente - die Aggressivität, die Moral, die Entrüstung bzw. ,indignatio' und die Negativität bzw. das ,Verkehrte' -, die das funktionale Feld der Satire beschreiben. ${ }^{12}$ Dies unterscheidet die Gattung von der Polemik, deren aggressiver Ironie die kritische Einbindung in den gesellschaftskritischen und somit moralischen Diskurs fehlt, sowie von der Groteske, in der die kritische und insofern referentielle Funktion aus strategischen bzw. wirkungsästhetischen Gründen ausgespart bleibt. ${ }^{13}$

9 Tschizewskij (1976) Satire, S. 278.

10 Gaier (1967) Satire, S. 336.

11 Vgl. Gaier (1967) Satire, S. 3.

$12 \mathrm{Vgl}$. Arntzen (2003) Satire.

13 Hinsichtlich dieser möglichen Deutung der Satire als Form einer Stellungnahme unterscheidet Könneker allerdings zwischen der nennenden bzw. gelenkten und der 


\section{Sarkasmus als Kennzeichen der invektiven Satire}

Ich betonte eingangs, dass sich die Satire als Rahmen anbietet, um invektives Sprechen bzw. Invektivität als literarische Form zu profilieren, insofern die Satire einen Grenzfall des Invektiven darstellt. Ein solcher Grenzfall wird in der einsetzenden Moderne dann ersichtlich, wenn wir den für invektives Sprechen wichtigen Begriff des Sarkasmus hinzuziehen. Denn seit Robert Burtons The Anatomy of Melancholy ist der Sarkasmus einerseits Widerpart der christlich-augustinischen Demutslehre, andererseits Merkmal der sich in der Renaissance aktualisierenden menippeischen Satire. Am Sarkasmus Gefallen zu finden, setzt daher die Ausblendung der klassizistischen Satiretheorie im Sinne Casaubons voraus. Dies zeigen die im England des siebzehnten Jahrhunderts einsetzenden Reflexionen über den Typus der , lustigen Person' als einer sarkastischen. In Edmund Gaytons Pleasant notes upon Don Quixot von 1654 wird Sancho Pansa als "Satyr" bzw. "Sarcast"14 charakterisiert, und schon in Edmund Spensers The Shepheardes Calender von 1579 prägt die Figur des Tom Piper "an Ironicall Sarcasmus, spoken in derision of these rude wits, whych make more account of a ryming rybaud, then of skill grounded upon learning and judgement." 15 Wie wichtig Burtons Sarkasmus-Kritik für die augusteische Satire-Poetik ist, zeigt die Diskussion darüber, wann in der Satire Formen der Beleidigung bzw. Diffamierung einer öffentlichen Person vorliegen. Man unterscheidet zwischen expliziter und impliziter Rede, d.h. zwischen der Schmähung (libel) und Beleidigung (slander) einerseits und den ironischen Formen ,buffoonery', ,jest', ,ridicule' und ,raillery' andererseits. Lässt sich der Tatbestand der Beleidigung anhand eines konkreten Vokabulars belegen, das in Handbüchern wie John Marchs Action for

darstellenden bzw. ungelenkten Satire: Während in der ungelenkten Satire der Autor in den Hintergrund tritt und dem Leser die Interpretation überlässt, versucht er in der gelenkten Satire, den Leser direkt zu überzeugen und zu belehren, wobei meist, wie in der Moralsatire, der Leser selbst das Ziel des satirischen Angriffs ist. Vgl. Könneker (1991) Satire, S. $15 f$.

14 Gayton (1654) Notes, S. 108.

15 Spenser (o.J.) Calender, S. 103.
Slander (1647) oder William Sheppards Action upon the Case of Slander (1674) aufgelistet ist, so ist der Nachweis weit komplizierter, wenn die Beleidigung ironisch eingekleidet ist. Für die neue Semantik des Sarkasmus ist dies von entscheidender Bedeutung, da er nun nicht allein als Trope, sondern zudem als literarisches Stilprinzip im Kontext der Satire diskutiert wird. Schon 1619 beschreibt Henry Huttons Spruchsammlung Follie's anatomie or satyres and satyricall epigramms die "satyres art" seiner Muse als "harsh sarcasmes, dissonant and smart"; 16 und noch hundert Jahre später heißt es in Anthony Blackwalls An Introduction to the Classics: "any keen Saying, which has the true point of Satyr, and cuts deep, is call'd a Sarcasm." ${ }^{17}$ Die Begriffe ,raillerie', ,ridicule' sowie ,buffoonery' dienen der Apologie der Satire, sie charakterisieren ihren ,wit' und unterscheiden sie somit vom ,lampoon' und dem ,libel', den literarisch anspruchsloseren Formen der Schmähschrift. ${ }^{18}$ Zur Unterscheidung dieser beiden Formen dient ein bei Cicero entwickeltes Merkmal: Das ,sal atticum', was als attisches Salz bzw. attischer Witz übersetzbar ist. Dass sich wesentliche Charakteristika der augusteischen Satire der Rezeption antiker Autoren - insbesondere natürlich Horaz und Juvenal - verdanken, verdeutlicht auch dieser Begriff des Witzes. Er impliziert eine Metaphorik, die schon in Ciceros 46 v. Chr. entstandener Schrift Brutus geprägt worden ist und den attischen vom asianischen Stil unterscheidet: „P. Scipio, qui est in consulatu mortuus, non multum ille quidem nec saepe dicebat, sed et Latine loquendo cuivis erat par et omnis sale facetiisque superabat." ${ }^{19}$ Mit Rückgriff auf Cicero werden auch die härteren Fälle der Personalsatire diskutiert, wie dies in Ben Jonsons Verteidigung der von Burton verworfenen Komödien des Aristophanes der Fall ist: "If all the salt in the old comoedy/ Should be so censur'd, or the sharper wit/ Of the bold satyre, termed scolding

16 Hutton (1619) Anatomie, S. 10. 17 Blackwall (1719) Introduction, S. 179.

18 Vgl. dazu Bredvold (1975) Note.

19 Cicero, Brutus 34,128. „Publius Scipio, der während seines Konsulats starb, pflegte zwar nicht viel und auch nicht gerade oft zu reden, aber mit seinem Latein war er jedem gewachsen und mit seinen scharfen, witzigen Pointen übertraf er alle." Übersetzung Kytzler (2000). 
rage,/ What age could then compare with those, for buffons?"20

Dass eine Satire auch bitter schmecken kann, wird durch den Vergleich mit der Galle ausgedrückt. Diese Assoziation geht zurück auf die Tradition der klassischen Verssatiren Théophiles de Viau, ${ }^{21}$ bleibt aber in den klassizistischen Apologien der augusteischen Satire ausgeschlossen: "not blunt and bitter rimes". ${ }^{22}$ Beschreibt das Salz den Witz, so die Galle den Zorn einer Satire; ist der salzige Witz durch das Adjektiv ,körnig' bestimmt, so bedient die Metapher der Galle den für den Sarkasmus zentralen Charakter der ,Bitterkeit'. Daneben steht das Motiv des ,MitEssig-Begießens', das auf Horaz zurückgeht. ${ }^{23}$ Es handelt sich also um geschmackliche Kriterien, denn es wird zwischen feinen und weniger feinen bzw. bitteren Formen des Witzes unterschieden. Am feinsten ist der urbane Witz, der sich durch Eleganz und Geist auszeichnet und der bäuerlichderben Form der Schmähschrift abgesprochen wird: „For Libel and true Satyr different be; This must have Truth, and Salt, with Modesty" ${ }^{24}$, so heißt es in Thomas Shadwells The medal of John Bayes von 1682. Während im Libel die Frechheit des Autors offenkundig ist - "[...] not his Wit, but Sawciness excels $[\ldots]^{\prime 25}$-, erweist sich der echte Satiriker als Erbe einer Tradition, in der es darum geht, durch den urbanen Witz das Laster statt der Person zu verlachen, so wie dies schon Horaz tat:

\begin{abstract}
Sparing the Persons, this does tax the Crimes, Call's not great Men, but Vices of the Times, With Witty and Sharp, not blunt and bitter rimes. Me thinks the Ghost of Horace there I see, Lathing this Cherry-cheek'd Dunce of Fifty Three; Who, at that age, so boldly durst profane, With base hir'd Libel, the free Satyr's Vein. ${ }^{26}$
\end{abstract}

Wenngleich der ,asianische Schwulst', auf den Ciceros Brutus reagiert, in der augusteischen Satire nicht zur Disposition steht, greift sie dennoch auf ebendiese Charakterisierung Ciceros

20 Zitiert nach Knox (1961) Irony, S. 191.

21 Vgl. Meyer-Minnemann (1969) Tradition, S. 92.

22 Shadwell (1682) Medal, S. 2.

23 Vgl. Horaz (2015) Sat. I,7,32.

24 "Denn Beleidigung und wahre Satire sind zweierlei; diese muß Wahrheit und Witz in Maßen besitzen". Shadwell (1682) Medal, S. 2.

25 Shadwell (1682) Medal, S. 1.

26 Shadwell (1682) Medal, S. 2. zurück, betont also den „Attic Wit"27 der satirischen Form. Und vor dem Hintergrund der durch Casaubon ausgelösten Satura-Satyr-Etymologie kann der Begriff, sarcastic' dann als Synonym für ,Satyrical' gesehen werden, wenn dieser über die Satyr-Figur oder die Satiren Juvenals identifiziert wird und nicht jene Orientierung an der auf Horaz bezogenen heiteren Satire vorliegt. Denn nur Horaz entbehrt des, beißenden' Spottes und schont sein Opfer; sein berühmtes, ridentem dicere verum' meint ja eine eher humorvolle Form des Verweisens auf Torheiten. Juvenal als Autor der strafenden Satire hingegen bedient sich eher beißender Ironieformen, denn inm geht es um ein ,entrüstetes' Verweisen auf die Lasterhaftigkeit von Personen. In diesem Sinne wird in John Drydens Discourse on the original and progress of Satire von 1693 die Differenz zwischen Horaz und Juvenal über den Salzgehalt ihrer Satiren bestimmt: "his wit", so schreibt Dryden über Horaz, "is faint; and his Salt almost insipit."28 Die Schärfe und Würze der Satire ist bei Dryden bezogen auf ihren sozialkritischen Gehalt: "the Sauce of Juvenal is more poignant", denn:

His thoughts are sharper, his Indignation against Vice is more vehement; his Spirit has more of the Commonwealth Genius; he treats Tyranny, and all the Vices attending it, as they deserve, with the utmost rigour. ${ }^{29}$

Der von John Dryden geprägte und später von Shaftesbury übernommene Begriff des, fine raillery' bezeichnet nun eine Technik des feinen Stichelns, die ihren Ursprung in der höfischen und zugleich korrigierenden Konversation hat, in der die Gesellschaft ihre Normen durchsetzt und bestätigt, ohne ihr ,Anderes' - den satirischen Gegenstand - durch das Lachen zu vernichten. Vielmehr wird dadurch die implizite Verspottung als genuine Kunstform der Satire anerkannt: "how hard to make a Man appear a Fool, a Blockhead, or a Knave, without any of those opprobrious terms!" 30 ,Raillery' unterscheidet die Satire von der Schmähschrift (,lampoon'), weil sie nicht explizit, sondern implizit lächerlich macht, was wiederum als Zeichen von Geist und Witz begrif-

27 Pope (1738) Epilogue, S. 317.

28 Dryden (1693) Discourse, S. 63.

29 Dryden (1693) Discourse, S. 65.

30 Dryden (1693) Discourse, S. 70. 
fen wird. Eben darin aber liegt der eigentliche konversationsgenehme bzw. gesellige Aspekt: "A witty Man is tickled while he is hurt in this manner; and a fool feels it not." 31 Unter Verweis auf sein eigenes Werk "Absalom and Achitopel" schreibt Dryden weiter:

The Character of Zimri in my Absalom, is, in my Opinion, worth the whole Poem: ,Tis not bloody, but ,tis ridiculous enough. And he for whom it was intended, was to witty to resent it as an injury. ${ }^{32}$

Dieser Begriff des, wit' wird später durch das von Theoretikern der Whigs wie Anthony Ashley Cooper, dem späteren Earl of Shaftesbury, eingeforderte satirische Konzept der ,politeness' bzw. des ,polite writings' abgelöst. Auch Autoren wie Richard Steele oder Joseph Addison fordern in den whiggistischen moralischen Wochenblättern The Tatler und The Spectateur ein am Paradigma des polite writing orientiertes Satirekonzept. In seinem Letter concerning Enthusiasm von 1708 durchleuchtet Shaftesbury zunächst die Gründe für jenes Unbehagen am Sarkasmus, wie es von Cicero über Augustinus bis hin zu Burton zu finden war. Es ist letztlich Ausdruck der Angst vor dem Wahrheitsgehalt des Spottes, die eben dann entsteht, wenn der Verspottete tatsächlich lächerlich ist. Den "test of ridicule", ${ }^{33}$ der in Shaftesburys Letter concerning Enthusiasm zur Unterscheidung des echten vom falschen Enthusiasmus präsentiert wird, bestehen nur jene Menschen, bei denen Sein und Schein keinen Widerspruch darstellen, denn sonst wirken sie schon von sich aus lächerlich. Anlass dieser Argumentation ist das Auftreten fanatischer französischer Protestanten in England. Die durch sie verursachten Störungen des öffentlichen Lebens sollten nicht durch obrigkeitliche Maßnahmen bekämpft werden; vielmehr sei Spott das geeignetste Mittel, den Fanatismus abzuwehren, denn Spott (,raillery') und Witz (,wit') enthüllten den falschen Ernst vorgeblicher Propheten und machten ihren Betrug offenbar. Shaftesburys Plädoyer: "Wit can never have it's liberty, where the freedom of Raillery is staken away" 34 ist daher im Grunde ein Einspruch gegen das Sarkasmus-

31 Dryden (1693) Discourse, S. 70.

32 Dryden (1693) Discourse, S. 71.

33 Shaftesbury (1708) Letter concerning Enthusiasm, S. 17. 34 Shaftesbury (1708) Letter concerning Enthusiasm, S. 31.
Verbot im Namen der geistreichen Geselligkeit einer Epoche. Echter Enthusiasmus ist demnach ein Zeichen für Witz und Geist, der sich auch auf die geistreiche Verspottung im Sinne des Begriffes ,raillery' versteht. Insofern ist, raillery' nicht diffamierend, sondern amüsant für denjenigen, der den "test of ridicule" zu bestehen vermag.

In seiner 1709 erschienenen Schrift Sensus Communis, die er einen Versuch über die Freiheit von Witz und Laune nennt, bestimmt Shaftesbury wie Cicero die "Urbanität" als "das rechte Maß", von der ein "possenhaftes, ungehobeltes Gebaren" zu unterscheiden sei. ${ }^{35}$ Das Argument dieser Schrift ist, dass der Freiheitsbegriff nicht allein auf die enge Programmatik republikanischer oder ständischer Theorien beschränkt werden könne, denn er beinhalte neben Tugenden wie Frugalität, Aufrichtigkeit und Offenheit auch den Witz. 'Politeness', formuliert Shaftesbury in seinem Programm, umfasse verfeinerten Geschmack, gewitzte Konversation und humorvoll-ironischen Umgang mit politischen wie religiösen Autoritäten in einer "common society". ${ }^{36}$ In Sensus Communis wird die von ,politeness' geprägte Satire zur Grundlage für eine möglichst radikale Form der Aufklärung und der Kritik. Die Formel „All politeness is owing to liberty" ${ }^{137}$ plädiert daher auch für die Freiheit von der Zensur, woraufhin sich notwendigerweise eine Verfeinerung des Witzes und des Umgangs entwickeln würde. Und wie bei Dryden so ist auch bei Shaftesbury Horaz das Paradigma des höflichen Schreibens, er ist "the most Gentleman-like of the Roman Poets". ${ }^{38}$ Neben der römischen Rhetorik ist die Satire des Horaz, inr Stil des, sermo urbanus', die zweite wichtige Quelle für Höflichkeit und Urbanität des Satirikers.

In Anthony Collins 1729 entstandenem Essay A discourse concerning Ridicule and Irony in writing wird der Begriff Sarkasmus in dezidiert affirmativer Form verhandelt. Er ist nicht länger eine wertneutrale Figur der Rhetorik, sondern wird als ambivalentes Mittel satirischen Schreibens begriffen und verteidigt. Collins kritisiert ebenso wie Shaftesbury die Praxis der Zensur, ${ }^{39}$ welche nicht

35 Shaftesbury (1992) Edition, S. 33: "the just measure of what we call Urbanity”; S. 32: "a Buffooning Rustick Air".

36 Shaftesbury (1709) Sensus Communis, S. 24.

37 Shaftesbury (1709) Sensus Communis, S. 8.

38 Shaftesbury (1710) Soliloquy, S. 169.

39 Vgl. Collins (1729) Discourse, S. 3. 
nur bei Verspottungen historischer Persönlichkeiten, sondern schon beim Spott auf das Zivil- und Kirchenrecht greift. Ein zeitgenössisches Argument gegen solche "controversial Writings" lautet: "municipal Laws, how trivial soever in their intrinsick Value, are never to be insulted; never to be treated with Buffoonery and Banter, Ridicule and sarcastick Irony." ${ }^{40}$ Collins argumentiert hingegen, dass gerade die Schriften orthodoxer Kirchenväter einschlägige Beispiele für ebenjene Form eines "controversial Writings" sind. Er erinnert daran, dass Erasmus von Rotterdams Colloquia familiari von 1518 in Anlehnung an Lukians satirische Dialoge entstanden ist, und verweist damit auf die Universalität sarkastischer Kommentare. Verspotten, Verlachen und Lächerlichmachen seien allgemeine Formen des Streitens, deren sich die Vertreter von Zivil- und Kirchenrecht - zu denen Erasmus selbst gezählt werden könne - ebenso bedienten wie ihre Gegner, die englischen Satiriker des 17. und frühen 18. Jahrhunderts:

Laughing therefore, and Ridicule in serious Matters, go round the World with no inconsiderable Applause, and seem highly proper for this World of Nonsense and Folly. To hinder laughing upon such Occasions as are given, is almost all one as to hinder breathing. ${ }^{41}$

Wenn Collins die Satire als "Ridicule in serious Matters" versteht, dann erklärt sich diese Betonung des Seriösen durch den an sich eher schärferen Charakter des Wortes. Die Begriffe korrespondieren dem in John Drydens Discourse concerning the Original and Progress of Satire von 1693 angestrengten Vergleich zwischen Horaz und Juvenal. Charakterisiert die humorvoll-ironische Kunst der ,raillery', die kleine persönliche Schwächen mit einem wohlwollenden Augenzwinkern aufs Korn nimmt, die Satiren Horaz', so steht der Begriff ,ridicule' für die weitaus aggressiveren Satiren Juvenals. Die Regeln der klassizistischen Poetik der Satire, wie sie in Drydens Abhandlung formuliert sind, schließen persönliche Verunglimpfung (,libel' oder ,lampoon') aus: Satire, so heißt es unter Bezug auf Dryden in Corbyn Morris' Essay towards Fixing the True Standarts of Wit, Humour, Satire, and Ridicule von 1744, "is a witty and severe Attack of mischievous Habits or Vices". ${ }^{42}$ Bezieht

40 Collins (1729) Discourse, S. 4.

41 Collins (1729) Discourse, S. 21.

42 Morris (1967) Essay, S. 36. sich also in dieser Definition des Begriffes ,Satire' die Verspottung ausschließlich auf Laster - "Spare then the Person and expose the Vice" ${ }^{43}$, so heißt es entsprechend in Alexander Popes 1738 erschienenen Satires and Epistels of Horace imitated -, so sind ,raillery' und ,ridicule' auch personenbezogen denkbar. Sie unterscheiden sich jedoch in der Härte ihres Spottes: "to avoid degrading the Person attack'd"44, ist die Maxime der ,raillery'; "to degrade the Person attack'd"45, ist die Maxime des ,ridicule'. In Eliza Haywoods Female Spectator von 1755 wird der Sarkasmus in ebendiesem Spannungsfeld verortet:

The difference between ridicule and raillery is so small, that the one is often mistaken for the other. - The latter, therefore, ought never to be attempted but by people of fine taste, nor played off but on those equally qualified to return it; and as it has also some distant affinity to satire, should never have for its subject matters of too serious a nature. - What exposes any thing we wish to have concealed, though it may be done with an air of pleasantry, leaves a sting behind it which is not easily forgiven, and will be taken for ridicule, wether meant as such or not. I know nothing in effect that sticks longer on the mind than a bitter sarcasm, especially when conscious of its having some foundation in truth. - But you will say this is not raillery. I grant it is ridicule, - it is invective; yet it is that which, with people of narrow understandings, passes for raillery, and as such is excused, if not applauded. ${ }^{46}$

\section{Die deutschsprachige Satire in der frühen Moderne}

Für die deutschsprachige Satire ist in diesem Zusammenhang ein Aspekt zu bedenken, der sie von der französischen und englischen Satire unterscheidet. Zum einen spielt die römische Verssatire aufgrund einer verspäteten Rezeption in der deutschen Literatur eine vergleichsweise geringe Rolle. Zum anderen fand die Diskussion darüber, was in der Satire erlaubt ist, aufgrund dieser verspäteten Rezeption der römischen Antike weitgehend ohne den Rekurs auf die römische Rhetorik und die damit verbundene Tradition der ironischen Tropen statt, in denen

43 Pope (1953) Imitations, S. 314.

44 Pope (1953) Imitations, S. 37.

45 Pope (1953) Imitations, S. 37.

46 Haywood (1771) Female Spectator, 7th Edition, S. 136. 
der Sarkasmus ein wichtiges Element darstellte. Dagegen findet man in der wichtigen Satiredefinition John Drydens eine Unterscheidung ironischer Formen, wie sie auch die antike Rhetorik kannte: die ,illusio' und die ,dissimulatio'. Aus dieser Differenz entfaltet sich in der zwischen Dryden und Shaftesbury stattfindenden Diskussion um die Freiheit des satirischen Witzes die Unterscheidung zwischen, ridicule' und ,raillery', die im Sinne der antiken Rhetorik feinere und gröbere Formen des satirischen Spottes bezeichnet. An die Stelle dieser Differenz tritt in der deutschen Diskussion über Ironie und Satire in der Romantik eine der antiken Rhetorik unbekannte Größe: der Humor. Und dieser unterscheidet nicht mehr graduell zwischen feineren und gröberen Formen, sondern kategorisch zwischen herzlich und herzlos.

Christoph Martin Wieland ist die zentrale Gelenkstelle. Er ist in Deutschland der eigentliche Importeur des Begriffes, Sarkasmus' und zugleich der letzte wirkliche Vertreter der antiken ,urbanitas'. Nach Wieland orientiert sich die für jede Satiretheorie wichtige Unterscheidung zwischen heraufsetzender und herabsetzender Komik nicht mehr an der antiken Rhetorik, sondern am Begriff des Humors. Wieland gewinnt sein durchaus kritisches Verständnis davon, was in der Satire erlaubt und verboten ist, aus der skizzierten alteuropäisch-republikanischen Tradition. Bezüge zu Shaftesbury finden sich auch bei Zeitgenossen wie Lichtenberg, Mendelssohn, Nicolai oder Lessing; wie wichtig jedoch die Rolle Wielands bei der Vermittlung dieser Tradition ist, zeigen Herders 1793-1797 veröffentlichte Briefe zur Beförderung der Humanität, in denen die "belehrende Schule der Urbanität" gelobt wird, die Christoph Martin Wieland durch seine Horaz-Übersetzung und -Kommentierung „jedem feineren Menschen [...] eröfnet" habe. ${ }^{47}$ Bei Wieland selbst klingt es so:

Dasjenige, was man in den schönsten Zeiten von Rom unter dem Wort Urbanität begriff, diesen Geschmack der Hauptstadt und diese feine Tinktur von Gelehrsamkeit, gehörig zu empfinden, setzt eine Menge Kenntnisse voraus, die auch dem gelehrtern Teile Weltkenntnis und Politesse [...], selbst diese Urbanität an einem Schriftsteller der Leser nicht allezeit gegenwärtig sind. ${ }^{48}$

47 Herder (1794) Briefe, 4. Sammlung, 51, S. 142. 48 Wieland 9 (1986) Übersetzung, S. 13.
Entstanden ist diese Einschätzung in einem "Hauptwerk" 49 Wielands: der Übersetzung und Erläuterung der Horazischen Episteln (erschienen 1782) und Satiren (erschienen 1786). Wichtig ist dieser Kommentar vor allem aus politischen Gründen, denn Wieland, der die Begeisterung seiner deutschen Zeitgenossen für die griechische Klassik nicht teilte, sondern "[s]eine Antike [...] erst später, mit dem vierten, dem philosophischen Jahrhundert und dem Hellenismus" beginnen und "im spätrepublikanischen und kaiserlichen Rom, im Zeitalter Ciceros, Horazens und Lukians"50 gipfeln lässt, findet in der Auseinandersetzung mit der römischen Antike eine gesellschaftlich-politische Utopie, den Republikanismus. In seinen Kommentaren ernennt Wieland Horaz zum "republikanische[n] Schriftsteller"51 schlechthin. Horaz, der als Offizier auf Seiten der Republikaner im Kampf gegen Oktavian erleben musste, wie "die Republik unter dem heftigsten Zweikampf zwischen Tyrannei und Freiheit [...] zu Trümmern ging", wird von Wieland zu den "Überbleibsel[n] einer bessern Zeit" gerechnet, zu den „wenigen vortrefflichen Köpfe[n], welche die Republik gesehen und überlebt hatten". ${ }^{52} \mathrm{Er}$ habe sich "die vollkommenste[...] Muße" bewahrt und "die Freiheit, mit sich selbst und für sich selbst zu leben", sogar im Umgang mit Augustus und Maecenas und ohne die Regeln der "feinste[n] Höflichkeit" zu verletzen. ${ }^{53}$ Diese „Kunst mit den Großen zu leben"54 habe Horaz beherrscht, sie sei das Signum seiner Urbanität: „Auf einem zugleich so schlüpfrigen und häkeligen Wege nie zu glitschen, ist vielleicht das Äußerste der Urbanität und des feinen Gefühls". 55

49 Reuter (1981) Die Philologie der Grazien, S. 280.

50 So Fuhrmann in seinem Kommentar zu Wieland, in: Wieland 9 (1986), S. 1081. Vgl. auch die Erläuterungen Wielands zum ersten Brief des Horaz: „Indessen ist doch nicht zu vergessen: daß [...] die Zeit, worin Cicero blühte, ganz eigentlich das schönste Alter der römischen Literatur war" (ebd., S. 408).

51 Reuter (1981), S. 278.

52 Wieland 9 (1986) Übersetzung, S. 297f. (Einleitung zum 19. Brief, 1. Buch).

53 Wieland 9 (1986) Übersetzung, S. 148f. (Einleitung zum 7. Brief, 1. Buch).

54 Wieland 9 (1986) Übersetzung, S. 277 (Einleitung zum 18. Brief, 1. Buch).

55 Wieland 9 (1986) Übersetzung, S. 743 (Einleitung zur 6. Satire, 1. Buch). 
Wenn Wieland am Beispiel des Horaz die Form der höflichen Satire, also "die gute Wirkung des zu rechter Zeit und am rechten Orte gebrauchten feinen Spottes, der Ironie", in eins setzt mit demjenigen, "was Shaftesbury das Licht des Lächerlichen nennt" 56 , bezeugt dies seine Kenntnis der englischen Satire-Theorien. Wielands Begriff der Satire rezipiert Shaftesbury, der im Letter concerning Enthusiam (1711) den "test of ridicule" einführte, bei dem Spott (,raillery') und Witz (,wit') den falschen Ernst der Schwärmerei enthüllen und diese vom echten Enthusiasmus unterscheidbar machen: ${ }^{57}$ Die Satire wird so zum "Probierstein der Wahrheit". ${ }^{58}$ Die satirischen Romane Wielands, z.B. sein Sieg der Natur über die Schwärmerey, oder die Abentheuer des Don Sylvio von Rosalva (1764) zeigen die Umsetzung dieser Theorie. Der Held muss von seiner Schwärmerei geheilt werden, um zur Tugend der Vernunft und damit zur Glückseligkeit gelangen zu können. In Wielands Spätwerk findet sich auch eine umfangreiche Phantasie zur griechischen Antike, und zwar in seinem "Liebling"59, dem fragmentarischen Briefroman Aristipp und einige seiner Zeitgenossen von 1800. Dieser fragmentarische Roman enthält schon im ersten Buch die gesamte Breite der bisherigen Ausführungen zu den antiken Formen der Ironie, zur Unterscheidung von ,illusio' und ,simulatio'. Wird im ersten Band die Ironie des Sokrates als Bestrebung begriffen, jemanden "mit dem ziemlich grobkörnigen attischen, oder vielmehr piräischen Salze seiner Sarkasmen tüchtig durchzureiben"60, so gelten im dritten Band des Aristipp die Komödien des Aristophanes als Ausdruck der "bittersten Sarkasmen über das Volk und die Regierung von Athen."61 Denn bei Aristophanes werde immer auch an das, Spott-Opfer Kleon' ${ }^{12}$ gedacht; gleiches gelte für die menippeische Satire Lukians, deren Opfer u.a. Sokrates gewesen sei. Wielands Fußnoten-Kommentar zu Lukians Der Verkauf der philosophischen Sekten

56 Wieland 9 (1986) Übersetzung, S. 831.

57 Verweyen (1979) Parodie, S. 80.

58 Nicolai (1799) Bildung, S. 80, zitiert nach Mollenhauer (1977) Satiren, S. 76.

59 Wieland über den Aristipp am 11. April 1803. Vgl. Sippwell (1988) Wielands "Liebling".

60 Wieland 33 (1800) Aristipp, S. 293.

61 Wieland 35 (1801) Aristipp, S. 8.

62 Wieland 35 (1801) Aristipp, S. 8. bedient sich dabei eines Arguments, das bereits für die augusteische Satire verbindlich war:

Die erste und wesentlichste Eigenschaft eines satirischen Werkes ist, daß dem Verspotteten kein Unrecht geschehe. Das Lächerliche muß in der Sache liegen, nicht vorsätzlich hineingebracht oder dem Belachten hinter seinem Rücken aufgeheftet werden. Witz und Laune können auch wohl bloßes Persiflage in einer fröhlichen Stunde unterhaltend machen: aber dann muß es wenigstens unschuldig sein. In diesem Aufsatze hat sich Lukian gegen die Philosophen alles erlaubt; Verdrehung und Verfälschung ihrer Lehrsätze, geflissentliche Mißdeutungen, elende Volkssagen und Märchen, kein Mittel ist ihm zu schlecht, um die größten und vortrefflichsten Männer aus dieser Klasse, selbst einen Pythagoras, Sokrates, Plato, Demokrit, Aristoteles, dem Spott eines ungelehrten Leserpöbels preiszugeben. [...] Die Billigkeit erfordert, zu erinnern, daß Lukian all diese Vorwürfe und Sarkasmen nicht aus dem Ärmel schüttelte, sondern mit Zeugnissen zu belegen imstande war. ${ }^{63}$

Wenn das Lächerliche „in der Sache" liegen muss, damit dem Opfer der Satire "kein Unrecht geschehe", dann beglaubigt auch Wieland die Maxime der augusteischen Satire: "Spare then the Person and expose the Vice." 64 Die Pikanterie eines satirischen Werkes, seine Würze, liegt also nicht allein im geistreichen bzw. ironisch-sarkastischen Gehalt, sondern ist zudem an ein moralisches Argument gebunden: Die Integrität der in der Satire attackierten Person muss bewahrt bleiben. Gilt diese Maxime nicht, dann verwandelt sich die Würze eines satirischen Werkes in einen bitteren Nachgeschmack.

\section{Nach der Rhetorik: Der deutsche Humor als Gegenbegriff zum sarkastischen Witz}

Mit der Romantik beginnt die Lösung von dieser in Rhetorik und Satire der alteuropäischen Klassik Englands und Frankreichs entwickelten Ironie. In Friedrich Schlegels romantischer Ironie ist die rhetorische Tradition nur noch auf den Bereich des

63 Wieland (1788), Lucians von Samosata Sämtliche Werke. Erster Theil, S. 363f. sowie S. 377.

64 Pope (1953) Imitations, S. 314. 
"Polemischen"65 beschränkt, der für das Konzept der progressiven Universalpoesie unwichtig ist, da es sich dabei um ein genuin literarisch-ästhetisches und nicht mehr politisch-rhetorisches Konzept handelt. Ironie im romantischen Sinne meint eine künstlerische Darstellungsform, die zur "Anschauung der absoluten Identität in der objektiven Totalität"66 führen soll. Sie gewinnt ihr Profil aus der Kritik an der Ich-Philosophie Fichtes, daher herrscht eine terminologische Verwandtschaft mit dem Begriff, intellektuelle Anschauung'. ${ }^{67}$ Schlegels Ironie bezeichnet ein (nachfichtesches) Schweben der Einbildungskraft. Zwar kennt Schlegel auch die Ironie im Sinne des sokratischen Dialogs, ${ }^{68}$ also die "besonnene Verstellung". ${ }^{69}$ Diese Ironie meint aber anders als bei Wieland keine hintergründige Spöttelei, sondern ermöglicht wie der Schlegel'sche Witz "echappées de vue ins Unendliche"70 durch das endlos poetische Schweben "zwischen dem Dargestellten und dem Darstellenden."71 Sie ist "klares Bewußtsein der ewigen Agilität"72, sie ist "innerste Mysterie der kritischen Philosophie"73; ein "universelles Experiment"74 und eine "Form des Paradoxon". ${ }^{75}$ Dieses Paradoxe meint die doppelte Reflexion

65 Schlegel (1967) Charakteristiken, S. 152: „Freilich gibt es auch eine rhetorische Ironie, welche sparsam gebraucht vortreffliche Wirkung tut, besonders im Polemischen; doch ist sie gegen die erhabne Urbanität der sokratischen Muse, was die Pracht der glänzendsten Kunstrede gegen eine Tragödie in hohem Styl."

66 Schelling (1797): Ideen zu einer Philosophie der Natur, S.73 sowie sinngemäß dazu Schlegel (1967) Charakteristiken, S. $182 \mathrm{f}$.

67 Im 448. Athenäum-Fragment heißt es: „Die intellektualen Anschauungen der Kritik sind das Gefühl von der unendlich feinen Analyse der griechischen Poesie und das von der unendlich vollen Mischung der römischen Satire und der römischen Prosa." In: Schlegel (1967) Charakteristiken, S. 254.

$68 \mathrm{Vgl}$. das berühmte 42. Lyceaumsfragment. In: Schlegel (1967) Charakteristiken, S. 152.

69 108. Lyceumsfragment. In: Schlegel (1967) Charakteristiken, S. 160 .

70 220. Athenäumfragment. In: Schlegel (1967) Charakteristiken, S. 200.

71 116. Athenäumfragment. In: Schlegel (1967) Charakteristiken, S. 182.

72 69. Ideenfragment. In: Schlegel (1967) Charakteristiken, S. 263.

73 Schlegel (1963) Philosophische Lehrjahre, S. 285.

74 Schlegel (1963) Philosophische Lehrjahre, S. 217.

75 48. Lyceumsfragment. In: Schlegel (1967) Charakteristiken, S. 153. des Subjekts, für welche Ironie der ästhetische Ausdruck ist. Begriffe wie Universalität - Unabschließbarkeit - Fragment identifizieren die Ironie als Beschränkung und "Idee eines Unendlichen"76, als "unendlich volles Chaos". ${ }^{77}$

Die Philosophie ist die eigentliche Heimat der Ironie, welche man logische Schönheit definieren möchte: denn überall wo in mündlichen oder geschriebenen Gesprächen, und nur nicht ganz systematisch philosophiert wird, soll man Ironie leisten und fordern; und sogar die Stoiker hielten die Urbanität für eine Tugend. Freilich gibts auch eine rhetorische Ironie, welche sparsam gebraucht vortreffliche Wirkung tut, besonders im Polemischen; doch ist sie gegen die erhabne Urbanität der sokratischen Muse, was die Pracht der glänzendsten Kunstrede gegen eine alte Tragödie in hohem Styl. Die Poesie allein kann sich auch von dieser Seite bis zur Höhe der Philosophie erheben, und ist nicht auf ironische Stellen begründet, wie die Rhetorik. Es gibt alte und moderne Gedichte, die durchgängig im Ganzen und überall den göttlichen Hauch der Ironie atmen. Es lebt in ihnen eine wirklich transzendentale Buffonerie. Im Innern, die Stimmung, welche alles übersieht, und sich über alles Bedingte unendlich erhebt, auch über eigne Kunst, Tugend, oder Genialität: im Äußern, in der Ausführung die mimische Manier eines gewöhnlichen guten italiänischen Buffo. ${ }^{78}$

In einer sehr hellsichtigen Notiz seiner Blütenstaub-Fragmente bemerkte Novalis dazu kritisch: „Was Fr. Schlegel so scharf als Ironie charakterisiert, ist meinem Bedünken nach nichts anderes als die Folge, der Charakter der Besonnenheit, der wahrhaften Gegenwart des Geistes. Schlegels Ironie scheint mir echter Humor zu sein."79 Darin zeigt sich neben einer Korrektur der Schlegel'schen Begriffsbildung auch die für die Fragestellung so wichtige Aufwertung des Humorbegriffs, die durch die Lösung des Ironiebegriffes von seinen rhetorischen Wurzeln vorbereitet wird. Wenngleich seine endgültige Definition als Signum einer ,Komik des Herzens' erst in den idealistisch-hegelianischen Ästhetiken des 19. Jahrhunderts stattfindet, so ist doch schon zu Novalis' Zeiten die Frontstellung antiker Ironiekonzepte zum ,Humor' erkennbar. Novalis' Kritik entwickelt sich zunächst vor dem Hinter-

76 Vgl. Schlegel (1969) Philosophische Vorlesungen, S. 357.

77 Vgl. Schlegel (1967) Charakteristiken, S. 263.

78 Vgl. Schlegel (1967) Charakteristiken, S. 151.

79 Novalis (1981) Blütenstaub, 29., S. 425. 
grund einer Übersetzungsschwierigkeit. Ging F. J. Riedel davon aus, dass der Humor eine Variante der alten ,urbanitas' sei - „Das attische Salz der Griechen und die Urbanität der Römer waren nichts anders, ein feiner Humour, womit sie ihre freundschaftlichen Zusammenkünfte würzten"80 -, so betont Johann Gotthelf Lindners Abhandlung Kurzer Inbegriff der Aesthetik, Redekunst und Dichtkunst von 1771:

Humor sagt mehr als der Griechen attisch Salz, das nicht sowohl den Charakter als vielmehr die Diction betrift, und mit Ironie einerlei sein kann, dergleichen in Lucian herrscht; mehr als der Römer Urbanität im Gegensatz der Rusticität. Beide nennt Riedel einen feinen Humour, zu Würze der Gesellschaften. Allein Humor ist mehr die individuelle eigenthümliche Form des Menschen aus sich selbst; Urbanität eine fremde nach Mustern der Gesellschaft, jedoch eine angenehme und gefällige. So ist auch Humor noch vom komischen Witz, den Sommerville Sneer nennt, von bloßer Ironie und Spötterey unterschieden. ${ }^{81}$

Wenn Lindner in seiner Polarisierung von Humor und Ironie die Humoristen zu den "launische Scribenten" 82 zählt, dann zeugt dies vom Begriffsverständnis des 16 . Jahrhunderts. Seit diesem findet man in der englischen Literatur die Bezeichnungen, good humour' und ,bad humour' und versteht darunter die Qualität der Säftemischung, welche eine gute oder schlechte körperliche und geistige Verfassung zur Folge hat. ,Humour' kann seit dieser Zeit ganz einfach mit Laune oder Stimmung übersetzt werden, sofern es mit einem qualifizierenden Attribut versehen ist. Analog dazu finden wir im Französischen die Unterscheidung von ,bonne humeur' und ,mauvaise humeur'. Gegen Ende des 16. Jahrhunderts bezeichnet man mit ,Humour' dann zunehmend ein abweichendes Verhalten von der gesellschaftlichen Norm, also Exzentrik, Manieriertheit und somit Lächerlichkeit, und schließlich den sich so gebenden Menschen selbst, der zur Zielscheibe des Spottes und der Satire wird. Dies geschieht im England des frühen siebzehnten Jahrhunderts in der Komödie Ben Jonsons, der sogenannten comedy of humours. Sie zeigt hervorstechende Schwächen der Menschen, indem die auftre-

80 Riedel (1967) Theorie, S. 94f.

81 Lindner (1971) Inbegriff, S. 158.

82 Lindner (1971) Inbegriff, S. 158. tenden Figuren auf exzentrische Weise einen bestimmten Charakterzug verkörpern: Jedermann auf seine Art, also Every man in his humour. In diesem Sinne kann John Dryden sagen: "Humour is the ridiculous extravagance of conversation, wherin one man differs from all others. "83 Als ein solcher Gegenstand erzeugt der Humor bei Dryden eine durchaus sarkastische Form des Komischen: "Wenn dieser Humor lebhaft und natürlich vorgestellt wird, so erzeugt er meistentheils das boshafte Vergnügen, welches sich durch das Lachen verräth, wie denn alle Abweichungen von dem Gewöhnlichen am geschicktesten sind, es zu erregen. "84 In diesem Sinne gelangte der Humorbegriff im 18. Jahrhundert als Lehnwort nach Deutschland. Entsprechend wurde anfangs die erste Silbe betont, eine allmähliche Akzentverschiebung fand erst später statt. Auch das Begriffsverständnis entspricht dem englischen, Lindners Unterscheidung zeigt dies: "Humor, Witz und Spötterey", so heißt es, "sind geschickte Nachahmungen ungereimter, widersinniger Charaktere". ${ }^{85}$ Auch Lessing und Herder verstehen Humor als Laune im Sinne des, bad or good humour'. Zieht man hingegen das 1788 erschienene Buch Über den Umgang mit Menschen von Adolph Freiherr von Knigge heran, dann lässt sich in diesem die heutige Bedeutung des Humors als einer versöhnlichen Komik des Herzens erkennen:

Wahrer Humor und ächter Witz lassen sich nicht
erzwingen, nicht erkünsteln; aber sie würken, wie das
Umschweben eines höheren Genius wonnevoll, erwär-
mend, Ehrfurcht erregend. Mit munteren, aufgeweck-
ten Leuten, die von ächtem Humor beseelt werden,
ist leicht und angenehm umzugehen. Ich sage, sie
müssen von ächtem Humor beseelt werden; die Fröh-
lichkeit muss aus dem Herzen kommen, muss nicht
erzwungen, muss nicht eitle Spassmacherey, nicht
haschen nach Witz seyn. ${ }^{86}$

Welche Konsequenzen diese Verabschiedung des klassischen Ironieverständnisses im Namen des Humors für den Status des sarkastischen Witzes hat, zeigt sich in Ansätzen schon in der Romantik. Man sucht eine beseeltere Form der Ironie. Schon

83 Dryden (1971) Essay, S. 59.

84 Dryden (1971) Essay, S. 61.

85 Lindner (1971) Inbegriff, S. 156.

86 Freiherr von Knigge (1788) Über den Umgang mit Menschen. 1. Teil, 1. Kapitel, 16, S. 61. 
Schlegel bedient sich kaum noch der für Wieland so wichtigen Kategorie der Urbanität, wenngleich er den Begriff sowohl in den Lyceums- und Athenäumsfragmenten als auch in seinen Aufsätzen Die Griechen und die Römer (1797) und Gespräch über die Poesie (1800) reflektiert. Er ersetzt dieses Wort jedoch durch Umschreibungen wie "Geselligkeit und römischen Witz"87, "Geselligkeit und gesellige Bildung" bzw. "Witz und gesellige Fröhlichkeit".88 Dies liegt an seiner geschichtsphilosophischen Unterscheidung zwischen antiker und moderner Literatur, ${ }^{89}$ Urbanität bezieht sich ausschließlich auf antike Literatur und wird anders als bei Wieland nicht auf die Moderne übertragen. Römische Urbanität lasse sich also einzig an den Satiren Horaz' ablesen: „Einheimisch war bei ihnen [den Römern] nur die Poesie der Urbanität, und mit der einzigen Satire haben sie das Gebiet der Kunst bereichert. [...] Die Satire gibt uns einen römischen Standpunkt für die Produkte des römischen Geistes." 90 Und eine vergleichbare Beschränkung auf die Antike erfährt nun auch der Begriff des Sarkasmus, der in Schlegels Athenäum ebenfalls auf den Geltungsbereich der Satire eingegrenzt wird:

Es gibt eine Art von Witz, den man wegen seiner Gediegenheit, Ausführlichkeit und Symmetrie den architektonischen nennen möchte. Äußert er sich satirisch, so gibt das die eigentlichen Sarkasmen. ${ }^{91}$

Was in der romantischen Ironie Friedrich Schlegels angedeutet ist, findet seine Fortsetzung in Jean Pauls 1804 verfasster Vorschule der Ästhetik sowie in der 1825 veröffentlichten Kleinen Nachschule zur ästhetischen Vorschule. Im Rahmen dieser Vorlesungsreihe über literarische Ästhetik unternimmt Jean Paul den Versuch, die Begriffe Humor, Komik, Witz und das Lächerliche eingehend zu beschreiben, gegeneinander abzugrenzen und zueinander in Beziehung zu setzen. Am Anfang steht die Distanzierung von der Hobbes'schen Deutung des Komischen als einem Resultat der Selbsterhebung, gegen welche Jean Paul im Anschluss an Schiller und Kant das Komische als "Genuß oder die Phan-

87 Schlegel (1979) Die Griechen und die Römer 1, S. 210 und: Die Griechen und die Römer 2, S. 296.

88 Schlegel (1967) Gespräch über die Poesie, S. 296.

89 Vgl. Jauß (1973) Replik.

90 Schlegel (1967) Charakteristiken, S. 296.

91 Schlegel (1967) Charakteristiken, S. 236. tasie und Poesie des ganz für das Freie entbundnen Verstandes" ${ }^{112}$ definiert. Resultat dieser idealistischen Umdeutung des Komischen ist der Begriff des Humors, der "als das umgekehrte Erhabene" nicht das Einzelne vernichtet, sondern das Große klein und das Kleine groß macht, also beides relativiert, "weil vor der Unendlichkeit alles gleich ist und nichts." ${ }^{193}$ Die aus dieser Formel resultierende Schwierigkeit, wie "der Humorist, welcher die Seele erwärmt"94, zu unterscheiden ist von einem ,Persifleur' wie Voltaire, führt zu einer Neudeutung der Begriffe Humor, Laune und Ironie, die folgenreich ist. Denn anders als Friedrich Schlegel unterscheidet Jean Paul den Humor "als wärmere und gleichzeitig umfassendere Art des dichterischen Ausdrucks" von der Ironie, die "als scharfe, kalte, intellektualistische Haltung" begriffen wird. ${ }^{95}$ Diese Unterscheidung stützt sich vor allem auf den Vergleich von Laurence Sterne und Jonathan Swift: "Sterne hat weit mehr Humor als Witz und Ironie; Swift mehr Ironie als Humor; Shakespeare Witz und Humor, aber weniger Ironie im engern Sinne."96 Durch diesen Rekurs auf Sterne wird dem Humorbegriff nun eine Form des narrativen Fiktionsbruches eingeschrieben, der dem romantischen Schweben zwischen Darstellung und Dargestelltem ähnelt, wie es in Friedrich Schlegels Begriff der romantischen Ironie ausgeführt ist. Im Unterschied zu Friedrich Schlegel wird in Jean Pauls Vorschule der Ästhetik der Sarkasmus nicht eigens behandelt. Er ist jedoch im Rahmen der diversen Formen des, romantisch Komischen' - Satire, Humor, Ironie, Laune und Witz - in seiner Bedeutung als beißender Witz in den Begriff der Ironie eingegangen. Denn steht die "vernichtende Idee des Humors" 97 im Zeichen der Dialektik des Unendlichen und Endlichen - Humor als das "umgekehrte Erhabene" 98 setzt das Endliche in den "Kontrast mit der Idee" 99 - und ist somit nach Behler ein der romantischen Ironie verwandtes Konzept, so führt die Identifikation Swifts als "ironischer Großmeister" 100 bei Jean Paul dazu, in

92 Paul (1963) Vorschule, S. 122.

93 Paul (1963) Vorschule, S. 125.

94 Paul (1963) Vorschule, S. 129.

95 So die Umschreibung Behlers, vgl. Behler (1996) Ironie/Humor, S. 827f.

96 Paul (1963) Vorschule, S. 144.

97 Vgl. Paul (1963) Vorschule, S. 124f.

98 Paul (1963) Vorschule, S. 125.

99 Paul (1963) Vorschule, S. 125.

100 Paul (1963) Vorschule, S. 150. 
der Ironie das sarkastische Indiz der Bitterkeit zu sehen: Hinsichtlich der "Bitterkeit der Ironie"101 ist "die swiftische nur darum die bitterste, weil sie die ernsteste ist." 102

Seit Jean Paul kann man sagen, dass es ein deutsches Wort Humor in der heutigen Bedeutung gibt. Mit inm wird eine Frontstellung von Humor und Ironie eingeführt, welche die Hegelianer in der Folge im Sinne einer moralischen Argumentation ausdeuten. Friedrich Theodor Vischers Ästhetik oder Wissenschaft des Schönen von 1846-57 begreift Ironie und Humor als die grundlegenden Faktoren des Komischen, den Humor jedoch als das umfassendere komische Vermögen. Über das objektiv Komische (Posse) und das subjektiv Komische (Ironie, Witz) hinausgehend, bringe der Humor das , absolut Komische' zum Ausdruck. Für den von Wieland eingeführten Begriff des Sarkasmus hat diese Argumentation freilich Konsequenzen: Dem sarkastischen Witz fehle das sogenannte ,Ethos des Lachens', wie es in Nicolai Hartmanns Ästhetik heißt. Ohne dieses Ethos als Zeichen des Humors ist Dichtung für Hartmann undenkbar, "darum ist der ,komische Dichter', der nur sarkastisch ist, wohl niemals dagewesen: seine Herzlosigkeit würde zum Himmel schreien"; genauer heißt es bei Hartmann:

In der Tat gibt es andere Arten, das Komische auszuwerten. [...] Von diesen Arten sind die wichtigsten:

1. das leere Amüsement am Komischen;

2. der Witz - die Auswertung der Komik zur Pointe;

3. die Ironie - das Geltendmachen der eigenen Überlegenheit durch scheinbare Herabsetzung des Ich; die Ablehnung in Form scheinbarer Anerkennung;

4. der Sarkasmus - die bittere, höhnende, vernichtende Ablehnung - in der Form übertriebener Anerkennung.

Die beiden letzteren sind dem Humor offenbar schroff entgegengesetzt. Denn der Humor behält immer auch als "grimmiger" - noch etwas Gutmütiges. [...] Der wirkliche Dichter des Komischen muß mehr haben als die Kunst, zu amüsieren, die Ironie, den Witz, den Sarkasmus. Er muß Humor haben. Und das heißt, er muß das höhere "Ethos des Lachens" haben, dasjenige, das nicht rein negativistisch eingestellt, nicht lieblos und herzlos ist, sondern aus der Fülle gemeinsamen Menschentums heraus sich auch noch mit dem Törichten und Kleinmenschlichen solidarisch fühlt und dem in aller mitreißenden Komik Ausdruck zu geben weiß. ${ }^{103}$

101 Paul (1963) Vorschule, S. 151.

102 Paul (1963) Vorschule, S. 151.

103 Hartmann (1953) Ästhetik, S. 420.
Man weiß seit den Arbeiten Preisendanz' um den Stellenwert des Humors für die Erzählkunst des 19. Jahrhunderts, ${ }^{104}$ und noch Thomas Mann betonte seine Freude darüber, dass man „in mir weniger einen Ironiker als einen Humoristen sieht." 105 Und dennoch hat wohl niemand die Absurdität des deutschen Humorbegriffes so präzise und zugleich so überaus ätzend auf den Punkt gebracht wie Karl Kraus in seinem 1921 erschienenen Essay Von Humor und Lyrik. Der Essay behandelt die „überwältigende Humorlosigkeit der deutschen Literatur"106, und diese These hat als wesentliche Voraussetzung, dass unklar bleibt, "was das Wesen des Humors ist, wenn inm der Witz fehlt." 107 Dies trifft nicht nur sehr präzise die zuvor erläuterte Entwicklung des Humorbegriffs im Deutschland zwischen Vormärz und Kaiserzeit. Kraus geht weiter, da er "an den großartigsten Beispielen von deutschem Humor"108 vor allem die „Eigenart" bemerkt, "keinen zu haben und für diese menschliche Schwäche ein verstehendes Lächeln aufzubringen. "109 Jean Paul ist bei Kraus neben den Xenien Goethes und Schillers das zentrale Beispiel dieser durch mangelnden Witz begründeten Humorlosigkeit:

Jean Paul, der gewiß in vielem verehrungswürdige und trotz umfassender Bildung unbeschränkte Geist, sagt, dass der Humor, als das umgekehrte Erhabene, nicht das Einzelne, sondern das Endliche durch den Kontrast mit der Idee vernichte; es gebe für ihn keine Toren, sondern nur Torheit und eine tolle Welt. Es wird wohl noch wenigen Lesern gelungen sein, an des Feldpredigers Schmelze Reise nach Flätz diese Erkenntnis zu überprüfen; aber ich glaube, dass der Witz unzweifelhaft daran festzustellen ist, dass er im Einzelnen das Endliche durch den Kontrast mit der Idee vernichtet, während der Humor eigentlich daran zu erkennen ist, dass er durch die Ausflucht in das Allgemeine dieses Kontrastes gar nicht habhaft und seine Beziehung auf die Idee oder seine Vernichtung des Endlichen nur glaubhaft wird, weil er nicht das Temperament hat, sich zu dem Einzelnen so herabzulassen, dass es nicht mehr vorhanden ist, was diesem doch widerfährt, wenn sich der Witz nur zu regen beginnt. ${ }^{110}$

104 Vgl. Preisendanz (1976) Humor.

105 Mann (1956) Humor, S. 167.

106 Kraus (1987) Humor, S. 199.

107 Kraus (1987) Humor, S. 205.

108 Kraus (1987) Humor, S.205.

109 Kraus (1987) Humor, S.205.

110 Kraus (1987) Humor, S. 205. 
Das Satirische als Grenzfall des Invektiven wird in der Moderne dann deutlich, wenn wir die begriffsgeschichtlich bzw. poetologisch sehr einschlägige Unterscheidung zwischen der strafenden und der heiteren Satire rekonstruieren. Denn diese seit dem 17. Jahrhundert bekannte und insbesondere bei Schiller aktualisierte Unterscheidung bleibt auch in der satirischen Praxis der Moderne eine leitende Orientierung. In dieser Moderne ist es jedoch die Unterscheidung zwischen humorvoller und sarkastischer bzw. invektiver Satire, die den moralischen Wert der Gattung als solcher markiert. Was der Satire erlaubt ist, wird dabei durch den Begriff des Humors definiert, was hingegen als Grenzfall gelten kann, zeigen Phänomene des literarischen Sarkasmus. Die in beiden Fällen wohl vorliegende Form einer "bitteren, höhnenden, vernichtenden Ablehnung" markiert jedoch nicht nur die Grenze zum satirischen Sprechen, sondern zugleich ebenjene Hürde, die der Satiriker in der Moderne zu nehmen hat, um provokant zu sein und sich Gehör zu verschaffen. Und wirft man von hier aus einen Blick auf die großen Satiriker der Moderne wie etwa Heinrich Heine, Kurt Tucholsky, Karl Kraus oder Hendryk Broder, dann wird schnell deutlich, dass Sarkasmus trotz dieser Grenzziehung zu den wichtigsten Ausdrucksformen des modernen Satirikers zu zählen sind. In dieser Funktion als Grenzfall des Invektiven liegt wohl die eigentliche Attraktivität des Satirischen für die moderne Literatur.

\section{Literaturverzeichnis}

\section{Quellen}

Adolph Freiherr von Knigge (1788): Über den Umgang mit Menschen. In zwey Theilen. Hannover: Schmidtsche Buchhandlung.

Blackwall, Anthony (1719): An Introduction to the Classics: Containing, A Short Discourse on their Excellencies; and Directions how to Study them on Advantage: With an Essay, on the Nature and Use of those Emphatical and Beautiful Figures which give Strength and Ornament to Writing. London.

Casaubon, Isaac (1605): De satyrica graecorum poesi et romanorum satira libri duo, Paris: Drouart.

Cicero (2000): Brutus: lateinisch - deutsch. 5. Auflage. $\mathrm{Hg}$. und übersetzt von Bernhard Kytzler. Düsseldorf/ Zürich: Artemis \& Winkler.

Collins, Anthony (1729): A discourse concerning Ridicule and Irony in Writing, in a letter to the Reverend Dr. Nathanael Marshall. Los Angeles. (=The Augustan Reprint Society; 142).
Cooper, Anthony Ashley, Third Earl of Shaftesbury (1708): A Letter Concerning Enthusiasm, to My Lord ****. London: J. Morphew.

Cooper, Anthony Ashley, Third Earl of Shaftesbury (1709): Sensus Communis: An Essay on the Freedom of Wit and Humour. London: E. Sanger.

Cooper, Anthony Ashley, Third Earl of Shaftesbury (1710): Soliloquy: or, Advice to an Author. London: J. Morphew.

Cooper, Anthony Ashley, Third Earl of Shaftesbury (1992): Sensus Communis: An Essay on the Freedom of Wit and Humour. In: Benda, Wolfram/Lottes, Wolfgang/ Uehlein, Friedrich A. et al. (Hgg.): Standard Edition. Sämtliche Werke, ausgewählte Briefe und nachgelassene Schriften. Bd. I.3. Stuttgart/Bad Cannstatt: Frommann-Holzboog Verlag.

Dryden, John (1971): An Essay of Dramatick Poesie (1668). In: Hooker, Edward N./Swedenberg, Hugh T./Roper, Alan T. et al. (Hgg.): The works of John Dryden XVII. Berkeley: University of California Press.

Dryden, John (1972): Poems 1681-1684. In: Hooker, Edward N./Swedenberg, Hugh T./Roper, Alan T. et al. (Hgg.) The works of John Dryden II. Berkeley: University of California Press.

Gayton, Edmund (1654): Pleasant notes upon Don Quixot. London: William Hunt.

Haywood, Eliza (1771): The Female Spectator. Vol. IV. $7^{\text {th }}$ Edition. London.

Hegel, Georg Wilhelm Friedrich (2013): Werke 14: Vorlesungen über die Ästhetik II. 9. Auflage. Hg. von Eva Moldenhauer. Frankfurt a. M.: Suhrkamp.

Herder, Johann Gottfried (1794): Briefe zur Beförderung der Humanität. 4. Sammlung. Riga: Hartknoch.

Horaz (2015): Satiren und Briefe. Lateinisch und deutsch. Nach der Übersetzung von Otto Schönberger, überarbeitet und mit Anmerkungen versehen von Friedemann Weitz. Darmstadt: WBG.

Hutton, Henry (1619): Folie's Anatomie or Satyres und Satyricall Epigrams. London.

Kraus, Karl (1987): Von Humor und Lyrik. In: Wagenknecht, Christian (Hg.): Die Sprache. Frankfurt a.M.: Suhrkamp.

Lindner, Johann Gotthelf (1971): Kurzer Inbegriff der Ästhetik, Redekunst und Dichtkunst (1771-1772). Bd. I. Frankfurt a.M.: Athenäum.

Mann, Thomas (1956): Humor und Ironie. Beitrag zu einer Rundfunkdiskussion. In: Ders. (Hg.): Nachlese. Frankfurt a.M.: Fischer, S. 166-169.

Morris, Corbyn (1947): An Essay towards Fixing the True Standarts of Wit, Humour, Raillery, Satire, and Ridicule. Los Angeles. (=The Augustan Reprint Society; 10).

Nicolai, Friedrich (1799): Ueber meine gelehrte Bildung, über meine Kenntniß der kritischen Philosophie und meine Schriften dieselbe betreffend, und über die Herren Kant, I. B. Erhard, und Fichte: Eine Beylage zu den neun Gesprächen zwischen Christian Wolf und einem Kantianer. Berlin/Stettin.

Novalis (1981): Blütenstaub. In: Ders.: Schriften. Zweiter Band: Das philosophische Werk I. Hg. von Richard 
Samuel. 3. Auflage. Stuttgart et al.: Kohlhammer, S. $412-474$.

Paul, Jean (1963): Vorschule der Ästhetik. In: Miller, Norbert (Hg.): Sämtliche Werke. Bd. 10. München: Carl Hanser Verlag.

Pope, Alexander (1969): Epilogue to the Satires. Written in 1738. Dialogue II. In: Butt, B. John (Hg.): The Poems of Alexander Pope. Imitations of Horace, Vol. IV. London: Methuen \& Co.

Quintilian (2000): Institutio oratoria 10. Übersetzt, kommentiert und mit einer Einleitung hg. von Franz Loretto. Stuttgart: Reclam.

Riedel, Friedrich Just (1767): Theorie der schönen Künste und Wissenschaften. Ein Auszug aus den Werken verschiedener Schriftsteller. Jena: Christian Henrich Cuno.

Schelling, Friedrich Wilhelm Joseph (1797): Ideen zu einer Philosophie der Natur als Einleitung in das Studium dieser Wissenschaft. In: Ders. (Hg.): Sämmtliche Werke. 1. Abteilung, Bd. 2. Stuttgart/Augsburg: Cotta'scher Verlag.

Schiller, Friedrich (1962): Sämtliche Werke. Bd. 5. München.

Schlegel, Friedrich (1963): Philosophische Lehrjahre I. In: Behler, Ernst (Hg.): Kritische Friedrich-SchlegelAusgabe 18: 2. Abteilung. München/Paderborn/Wien: Schöningh.

Schlegel, Friedrich (1967): Charakteristiken und Kritiken I. In: Eichner, Hans (Hg.): Kritische FriedrichSchlegel-Ausgabe. 2. Bd. (1796-1801). München/ Paderborn/Wien: Schöningh.

Schlegel, Friedrich (1969): Philosophie des Lebens, in 15 Vorlesungen gehalten zu Wien im Jahre 1827 und Philosophische Vorlesungen, insbesondere über Philosophie der Sprache und des Wortes, geschrieben und vorgetragen zu Dresden im Dezember 1828 und in den ersten Tagen des Januars 1829. In: Behler, Ernst (Hg.): Kritische Friedrich-Schlegel-Ausgabe 10: 1. Abteilung. Paderborn/Wien: Schöningh.

Schlegel, Friedrich (1979): Die Griechen und die Römer. In: Behler, Ernst (Hg.): Kritische Friedrich-SchlegelAusgabe 1: 1. Abteilung, Kritische Ausgabe Studien des klassischen Altertums. Paderborn: Schöningh.

Shadwell, Thomas (1682): The medal of John Bayes. London.

Spenser, Edmund (o. J.): The Shepheardes Calender. Conteyning twelue Aeglogues proportionable to the twelue monethes. In: de Sélincourt, Ernest (Hg.): Spenser's Minor Poems. Oxford: At the Clarendon Press.

Wieland, Christoph Martin (1788): Lucians von Samosata Sämtliche Werke. Aus dem Griechischen übersetzt und mit Anmerkungen und Erläuterungen versehen von C.M. Wieland. Erster Theil. Leipzig.

Wieland, Christoph Martin (1986): Bd. 9: Übersetzung des Horaz. Kommentar von Manfred Fuhrmann. Frankfurt a.M.: Suhrkamp.

Wieland, Christoph Martin (1800): Aristipp 1. Band. In: Ders. (Hg.): Sämmtliche Werke 33. Bd. Leipzig.
Wieland, Christoph Martin (1801): Aristipp 3. Band. In: Ders. (Hg.): Sämmtliche Werke 35. Bd. Leipzig.

\section{Forschungsliteratur}

Arntzen, Helmut (2003): Satire. In: Barck, Karlheinz/ Fontius, Martin/Schlenstedt, Dieter et al. (Hg.): Ästhetische Grundbegriffe. Bd. 5. Postmoderne bis Synästhesie. Stuttgart/Weimar: Metzler, S. 345-364.

Becker-Cantarino, Barbara (1985): Zur Satire in der deutschen Literatur der frühen Neuzeit. In: Daphnis 14, S. 605-613.

Behler, Ernst (1996): Ironie/Humor. In: Ricklefs, Ulfert (Hg.): Das Fischer Lexikon Literatur. Bd. 2. Frankfurt a.M: S. Fischer, S. 827-828.

Bredvold, Louis (1975): A Note in Defence of Satire. In: Fabian, Bernhard (Hg.): SATURA. Ein Kompendium moderner Studien zur Satire. Hildesheim/New York: Olms, S. 83-94.

Brummack, Jürgen (1977): Satire. In: Kohlschmidt, Werner/Mohr, Wolfgang (Hgg.): Reallexikon der deutschen Literaturgeschichte. Bd. 3. Berlin/New York: De Gruyter, S. 601-614.

Brummack, Jürgen (1971): Zu Begriff und Theorie der Satire. In: Deutsche Vierteljahrsschrift für Literaturwissenschaft und Geistesgeschichte 45, S. 275-377.

Gaier, Ulrich (1967): Satire. Studien zu Neidhart, Wittenwiler, Brant und zur satirischen Schreibart. Tübingen: Max Niemeyer.

Hartmann, Nicolai (1953): Ästhetik. Berlin: De Gruyter. Jauß, Hans Robert (1973): Schlegels und Schillers Replik auf die "Querelle des Anciens et des Modernes". In: Ders. (Hg.): Literaturgeschichte als Provokation. Frankfurt a.M.: Suhrkamp, S. 67-106.

Knox, Norman (1961): The Word Irony and its Context, 1500-1755. Durham (North Carolina): Duke University Press.

Könneker, Barbara (1991): Satire im 16. Jahrhundert: Epoche - Werk - Wirkung. München: Beck.

Meyer-Minnemann, Klaus (1969): Die Tradition der klassischen Satire in Frankreich. Themen und Motive in den Verssatiren Théophiles de Viau. Bad Homburg/ Berlin/Zürich: Gehlen.

Meyer-Sickendiek, Burkhard (2007): Satire. In: Ueding, Gert (Hg.): Historisches Wörterbuch der Rhetorik. Bd. 8. Tübingen: Niemeyer, S. 447-469.

Meyer-Sickendiek, Burkhard (2009): Was ist literarischer Sarkasmus? Ein Beitrag zur deutsch-jüdischen Moderne. München: Wilhelm Fink Verlag.

Mollenhauer, Peter (1977): Friedrich Nicolais Satiren. Ein Beitrag zur Kulturgeschichte des 18. Jahrhunderts. Amsterdam: John Benjamins Publishing Company.

Preisendanz, Wolfgang (1985): Humor als dichterische Einbildungskraft. Studien zur Erzählkunst des poetischen Realismus. München: Wilhelm Fink Verlag.

Reuter, Hans-Heinrich (1981): Die Philologie der Grazien. Wielands Selbstbildnis in seinen Kommentaren der Episteln und Satiren des Horaz. In: Schelle, Hansjörg (Hg.): Christoph Martin Wieland. Darmstadt: WBG, S. 251-306. 
Sippwell, Armin P. (1988): Wielands „Liebling”. Zur Genese des Romans „Aristipp und einige seiner Zeitgenossen". Colloquia Germanica 21, S. 99-110. Tschizewskij, Dimitri (1976): Satire oder Groteske? In: Preisendanz, Wolfgang/Warning, Rainer (Hgg.): Das Komische München: Wilhelm Fink Verlag, S. 269-278.
Verweyen, Theodor/Witting, Gunther (1979): Die Parodie in der neueren deutschen Literatur. Eine systematische Einführung. Darmstadt: Wissenschaftliche Buchgesellschaft. 